\section{Polymyalgia rheumatica following intravesical bacillus Calmette-Guerin instillation: coincidence or true association? A case report and literature review}

\section{Ciro Manzo}

Azienda Sanitaria Locale Napoli 3 sud, Sant'Agnello, Napoli, Italy

\begin{abstract}
We report the case of a 73-year-old male patient suffering from non muscle invasive bladder cancer (NMIBC) who had violent pains in his neck and shoulders associated with general discomfort and fever, following the second intravesical instillation of bacillus Calmette-Guerin (BCG), with further worsening after the third instillation. During his hospitalization, laboratory tests showed a significant raise of inflammatory markers. An ultrasound (US) examination of his shoulders showed bilateral longhead-biceps exudative tenosynovitis and subdeltoid bursitis. An 18-fluorodeoxyglucose positron emission tomography (18-FDG PET) associated with total body computed tomography (CT) showed pathological inflammatory findings in neck and shoulders, with exclusion of pathological findings in other sites. Cystoscopy was negative for NMIBC recurrence. Polymyalgia rheumatica (PMR) was diagnosed and BCG instillations was stopped. The patient had fast improvement of clinical manifestations and laboratory tests, but when he resumed them a few weeks later, the same manifestations recurred.
\end{abstract}

\section{Introduction}

Polymyalgia rheumatica (PMR) is considered the most frequent inflammatory rheumatic disease in Caucasians over the age of 70 years. ${ }^{1-3}$ Its diagnosis is based upon recognition of a clinical syndrome consisting of pain localized to neck and shoulder and pelvic girdles, associated with morning stiffness of inflammatory type (i.e. lasting more than 45 minutes). In some PMR patients, constitutional manifestations such as weight loss, fever of unknown origin, general discomfort, fatigue, and loss of appetite complete the clinical picture. ${ }^{4,5}$ To date, no specific laboratory tests are available. Erythrocyte sedimentation rate (ESR) and C-reactive protein (CRP) concentrations are usually raised at the time of diagnosis, but normal ESR and CRP should not be a reason of exclusion for PMR. ${ }^{6,7}$ Shoulder and hip ultrasound (US) examinations can give important contributions, as proposed by 2012 EULAR/ACR classification criteria. This EULAR/ACR collaborative initiative also highlighted that prednisone response is not reliable as a classification feature for PMR. ${ }^{8}$ The etiopathogenesis of PMR is still debated. Some infectious and environmental agents have been suggested as triggers, but data are mostly anecdotal. ${ }^{9}$

Intravesical instillation of bacillus Calmette-Guerin (BCG) after transurethral resection (TUR) is considered the goldstandard treatment for non-muscle invasive bladder cancer (NMIBC), at high risk of recurrence or progression. ${ }^{10} \mathrm{BCG}$ attachment to fibronectin present at the endoluminal layer, its internalization into resident immune cells, normal cells, and tumour urothelial cells, and the next induction of innate and tumour-specific immunity, are significant steps of this immunotherapy. The final result is the destruction of the neoplastic cells. ${ }^{11}$

\section{Case Report}

In 2015, a 73-year-old male patient affected with NMIBC complained of violent pains in his neck and shoulders associated with general discomfort and fever, after the second endovesical instillation of BCG. The intake of acetominophen determined fever resolution, and transient relief of pain. After the third instillation, pains in scapular girdles and neck worsened to the point that he was totally dependent on family members in daily life activities. During hospitalization, the laboratory tests showed: ESR = $52 \mathrm{~mm} / \mathrm{h}$; CRP concentration $=30 \mathrm{mg} / \mathrm{dL}$ (normal value $<6$ ). In the normal ranges were: serum fibrinogen levels, rheumatoid factor (RF) and anti-protein citrullinated antibodies (APCA), hemoglobin, transaminases, creatine phospho kinase, protein electrophoresis, antinuclear cytoplasmic antibodies (ANCA). Urinoculture and urine blood test were negative. Occult blood research in the stool was negative and fecal calprotectin dosage was in its normal range. Antibodies to hepatitis $\mathrm{C}$ virus and Australia antigen were absent. Cystoscopy showed normal findings. An US examination showed bilateral long-head-biceps exudative tenosynovitis and subdeltoid bursitis in his shoulders. 18-FDG PET/CT showed inflammatory findings in neck and
Correspondence: Ciro Manzo, Azienda

Sanitaria Locale Napoli 3 sud,

Poliambulatorio Distretto Sanitario 59, Viale dei Pini 1, 80065 Sant'Agnello, Napoli, Italy. E-mail: manzoreumatologo@libero.it

Key words: Polymyalgia rheumatica; bacillus Calmette-Guerin; non muscle invasive bladder cancer; immunotherapy

Conflicts of interest: the author declares declare no conflict of interest.

Ethical approval: the ethics committee of the Health District no. 59 gave ethical approval.

Informed consent: a written informed consen for publication was obtained from the patient The author declares full availability to show this informed consent, according to Italian privacy policy.

Received for publication: 10 February 2020. Accepted for publication: 24 March 2020.

This work is licensed under a Creative Commons Attribution-NonCommercial 4.0 International License (CC BY-NC 4.0).

${ }^{\circ}$ Copyright: the Author(s), 2020

Licensee PAGEPress, Italy

Geriatric Care 2020; 6:8868

doi:10.4081/gc.2020.8868

shoulders, with exclusion of pathological findings in other sites. According to 2012 ACR/EULAR criteria (total score of 8 in our patient), PMR was considered. His urologist advised to stop cautiously BCG endovesical instillations. In just a few days, the patient had a rapid improvement of pain and restored full autonomy. ESR and CRP normalized after 10 and 3 days, respectively. When he resumed the endovesical instillations of BCG a few weeks later, the same manifestations recurred and it was decided to stop them definitively.

During a follow up of 4 years, all PMRmimicking diseases were excluded (Table 1), and no alternative diagnosis was possible. Temporal artery color duplex sonography (TA-CDS), 18-FDG-PET with total body contrast-enhanced computed tomography (CT) and cystoscopy showed normal findings every time when the study was conducted.

\section{Discussion}

An involvement of joints, with the clinical pattern of reactive arthritis, is a wellknown side effect of intravesiscal BCG immunotherapy. By far, the large joints of lower limbs are the most involved and the 
Table 1. Main diseases with which PMR is in differential diagnosis, and signs and symptoms useful for a correct diagnosis.

\begin{tabular}{ll} 
Disease & Signs and symptoms useful for a correct diagnosis \\
Rheumatoid arthritis & $\begin{array}{l}\text { Involvement of some joints of the hands (metacarpophalangeal II and III, and proximal } \\
\text { interphalangeal), positive results of rheumatoid factor and anti-cyclic citrullinated peptide } \\
\text { antibodies (ACPA), radiographic and ultrasound findings (erosive arthritis, periarticular } \\
\text { osteoporosis) } \\
\text { Symmetric multiple synovitis, seronegative in rheumatoid factor and ACPA, causing boxing-glove } \\
\text { Remitting Seronegative Simmetrical Synovitis with }\end{array}$ \\
$\begin{array}{ll}\text { Pitting Edema (RS3PE) } \\
\text { tendon sheath }\end{array}$ \\
\hline Late-onset spondyloarthropathies, including ankylosing & Inflammatory pain in the lumbar region; radiographic findings of sacroiliitis; psoriasis
\end{tabular}

spondylitis and psoriatic arthritis

Late-onset systemic lupus erythematosus, sclerodermia, Inflammatory pain in the lumbar region; radiographic findings of sacroiliitis; psoriasis Sjogren's syndrome, vasculitis

Late-onset systemic lupus erythematosus, sclerodermia, Sjogren's syndrome, vasculitis

Idiopathic inflammatory myopathies

(dermatomyositis, polymyositis)

Scapulohumeral periarthritis, adhesive capsulitis (frozen shoulder)

Calcium pyrophosphate deposition disease

Presence of antinuclear antibodies, presence of antineutrophil cytoplasmic antibodies

Paraneoplastic syndromes

Skin rashes, increased creatine kinase in the blood

Restriction of shoulder movements, even in passive; ultrasound and magnetic resonance imaging allow one to diagnose the specific inflammation. Inflammatory markers not raised Monoarthritis; radiographic and ultrasound findings

Failure to respond to glucocorticoid therapy or frequent relapses must be considered as elements of suspicion. Furthermore, the presence of untypical clinical manifestations and of laboratory findings (among these, macrocytic anemia or bicytopenia), and familiarity for neoplasms should also be considered as warning

Fibromyalgia Inflammatory indices in their normal range, presence of tender points, widespread chronic pain

Reactive arthritis

Inflammatory back pain, additive or migratory oligoarthritis, presence of extra-articular symptoms, following a gastrointestinal or urogenital infection by a minimum of 1 to a maximum of 3-6 weeks. Long-term corticosteroid therapy is not required

asymmetry is the prevalent pattern. Arthritis usually has a self-limiting course with poor tendency to chronicity. The involvement of shoulder and pelvic girdles, typical of PMR, has never been described during BCG-reactive arthritis. ${ }^{12}$ A definite genetic link is documented by human leucocyte antigens (HLA) B27 carriers in about half cases, supporting the hypothesis that, at least in these patients, the failure of immunological tolerance in association with the presence of BCG in the bladder can lead to an immunomediate inflammatory reaction in the joints. ${ }^{13}$

Quite recently, we reported the case of a 69-year-old male patient suffering from PMR and remitting seronegative symmetrical synovitis with pitting edema (RS3PE), which occurred after a cycle of six intravesical instillation of BCG. In this patient, prednisone therapy was necessary for full resolution of PMR. ${ }^{14}$

According to literature review, only a case of PMR associated with giant cell arteritis $(\mathrm{GCA})^{15}$ and a case of isolated $\mathrm{GCA}^{16}$ have been reported. PMR and GCA are closely related and often overlapping conditions. In some patients, PMR may be the presenting manifestation of GCA. In addition to this, some investigators speculated that PMR might be an incomplete form of GCA, manifested in the proximity of axillary, subclavian, and/or femoral arteries. ${ }^{17}$ In our patient, diagnosis of GCA was clinically excluded at the time of diagnosis of PMR, and during follow-up using TACDS and 18-FDG PET/CT imaging.

The possibility that PMR may be a paraneoplastic syndrome has been widely discussed in the literature, with contrasting point of views. Bladder cancer can be diagnosed in the first year after the diagnosis of PMR. ${ }^{18,19}$ Therefore, the possibility that in our patient PMR could be a paraneoplastic finding was carefully excluded.

As for today, the reasons why in our patient PMR followed BCG immunotherapy for NMIBC are only speculative. According to the so-called molecular mimicry theory, the shared homology between BCG proteins such as heat-shock protein HSP65 and juxtasynovial proteins located in shoulder and/or pelvic girdles could play a relevant role. Genetic factors, related to major histocompatibility complex (MHC) class I, could be another favoring factor, acting as restriction molecules for antigenic bacterial peptides presented to and cross recognized by cytotoxic CD8+ T lymphocytes. Furthermore, these peptides possess a potential to skew the immune response toward Th1-like patterns. ${ }^{20}$ The senescence of the immune system as demonstrated by the loss of CD28 on CD4+ T senescent cells may be an additional responsible factor in patients with PMR, leading to aberrant immune responses. ${ }^{9}$

\section{Conclusions}

According to literature review, the occurrence of PMR during BCG intravescical insillations is very rarely described, despite the widespread use of this immunotherapy.

In our patient, its rapid disappearance after BCG instillations and its prompt reappearance after their re-introduction suggest that PMR has been a true association with this immunotherapy, and not a coincidence.

\section{References}

1. Partington R, Helliwell T, Muller S, et al. Incidence, prevalence and treatment burden of polymyalgia rheumatica in the UK over two decades : a population 
study. Arthritis Res Ther 2018;20:258.

2. Crowson CS, Matteson EL. Contemporary prevalence estimates for giant cell arteritis and polymyalgia rheumatica, 2015. Semin Arthritis Rheum 2017;47:253-6.

2. Manzo C. Incidence and prevalence of polymyalgia rheumatica (PMR): the importance of the epidemiological context. The Italian case. Med Sci (Basel) 2019;7:pii:E92.

3. Milchert M, Brzosko M. Diagnosis of polymyalgia rheumatica usually means a favourable outcome for your patient. Indian J Med Res 2017;145:593-600.

4. Gonzalez-Gay MA, Matteson EL, Castaneda S. Polymyalgia reumatica. Lancet 2017;390:1700-12.

5. Manzo C, Milchert M, Natale M, Brzosko M. Polymyalgia rheumatica with normal values of both erythrocyte sedimentation rate and C-reactive protein concentration at the time of diagnosis. Rheumatology (Oxford) 2019;5: 921-3.

6. Manzo C, Milchert M. Polymyalgia rheumatica with normal values of both erythrocyte sedimentation rate and $\mathrm{C}$ reactive protein concentration at the time of diagnosis: A four-point guidance. Reumatologia 2018;56:1-2.

7. Dasgupta B, Cimmino MA, Kremers HM, et al. 2012 provisional classifica- tion criteria for polymyalgia rheumatica: A European League Against Rheumatism/American College of Rheumatology collaborative initiative. Arthritis Rheum 2012;64:943-54.

8. Guggino G, Ferrante A, Macaluso F, et al. Pathogenesis of polymyalgia rheumatica. Reumatismo 2018;70:10-7.

9. Babjuk M, Bohle A, Burger M, et al. EAU guidelines non- muscle-invasive urothelial carcinoma of the bladder : update 2016. Eur Urol 2017;71:447-61

10. Pettenati $C$, Ingersoll MA. Mechanisms of BCG immunotherapy and its outlook for bladder cancer. Nat Rev Urol 2018; 15: 615-25.

11. Liu Y, Lu J, Huang Y, Ma L. Clinical spectrum of complications induced by intravesical immunotherapy of bacillus Calmette-Guerin for bladder cancer. J Oncol 2019;3.

12. Bernini L, Manzini CU, Giuggioli D, et al. Reactive arthritis induced by intravesical BCG therapy for bladder cancer: our clinical experience and systematic review of the literature. Autoimmun Rev 2013;12:1150-9.

13. Genereau T, Koeger AC, Chaibi P, Bourgeois P. Polymyalgia rheumatica with temporal arteritis following intravesical Calmette-Guerin bacillus immunotherapy for bladder cancer. Clin Exp Rheumatol 1996;14:110.
14. Stavris C, Retornaz F, Charpin C, et al. Vascularites des gros vaisseaux induites par une prise médicamenteuse: 2 nouveaux cas secondaires à l'administration de BCG-thérapie et de G-CSF. Rev Med Int 2016;37:A179.

15. Marzo-Ortega $\mathrm{H}$, McGonagle $\mathrm{D}$, O'Connor P, et al. Subclinical vasculitis in polymyalgia rheumatica. Ann Rheum Dis 2001;60:1058-9.

16. Manzo C. Polymyalgia rheumatica and remitting seronegative symmetrical synovitis with pitting edema following intravesical instillation of bacillus Calmette-Guerin. Reumatologia 2019; 57:249-52.

17. Manzo C, Natale M. Polymyalgia rheumatica and cancer risk: the importance of the diagnostic set. Open Access Rheumatol 2016;8:93-5.

18. Muller S, Hider S, Helliwell T, et al. The real evidence for polymyalgia rheumatica as a paraneoplastic syndrome. Reumatismo 2018;70:23-34.

19. Pahari S, Deepyan Chatterjee D, Negi $\mathrm{S}$, et al. Morbid sequences suggest molecular mimicry between microbial peptides and self-antigens: a possibility of inciting autoimmunity. Front Microbiol 2017;8:1938. 